\title{
Expression of cluster of differentiation 74 in gallbladder carcinoma and the correlation with epithelial growth factor receptor levels
}

\author{
PENG WANG ${ }^{*}$, QIAO SHI*, TENG ZUO, XIAOBO HE, JIA YU and WEIXING WANG \\ Department of Hepatobiliary and Laparoscopic Surgery, Renmin Hospital of Wuhan University, \\ Wuhan, Hubei 430060, P.R. China
}

Received December 21, 2014; Accepted January 22, 2016

DOI: $10.3892 / \mathrm{ol} .2016 .4191$

\begin{abstract}
Cluster of differentiation 74 (CD74), a transmembrane glycoprotein, has been previously reported to be important in the pathogenesis of several cancers, including hematological malignancies and solid tumors. The present study analyzed the significance of CD74 in gallbladder carcinomas (GBCs) and indicated the correlation of CD74 expression with epithelial growth factor receptor levels. Immunohistochemistry (IHC) was used to examine the expression of CD74 in GBC and normal gallbladder tissues, and western blotting was used to investigate whether CD74 expression varied in well-, moderately- and poorly-differentiated tumors. The correlation between the expression of CD74 and epithelial growth factor receptor levels was studied using the Spearman's rank correlation coefficient. The results of the IHC analysis revealed that CD74 was not expressed in the normal gallbladder tissues, and the mean integrated optical density value of CD74 in the poorly-differentiated tissues was increased compared with that in the well- and moderately-differentiated tissues. The results of the western blotting were consistent with the results of the IHC. The expression of CD74 was positively correlated with epithelial growth factor receptor levels $(r=0.607 ; \mathrm{P}<0.05)$. These results indicate that CD74 may be important in the progression of GBC.
\end{abstract}

\section{Introduction}

Gallbladder carcinoma (GBC) is a rare disease among the gastrointestinal cancers; however, GBC is a common malignant tumor in the extrahepatic biliary tract system, accounting for $80-95 \%$ of biliary tract cancers $(1,2)$. The morbidity rate

Correspondence to: Professor Weixing Wang, Department of Hepatobiliary and Laparoscopic Surgery, Renmin Hospital of Wuhan University, 99 Ziyang Road, Wuhan, Hubei 430060, P.R. China

E-mail: sate.1lite@163.com

*Contributed equally

Key words: gallbladder carcinoma, cluster of differentiation 74, epithelial growth factor receptor of GBC has markedly increased due to the advancing age of patients, and the increased rate of cholelithiasis and chronic inflammation (3). As GBC lacks a specific clinical presentation, the disease is usually diagnosed at the advanced stages, or is incidentally identified during or following a cholecystectomy (3). Despite the lack of remarkable clinical features, the unique anatomical features of the gallbladder, including abundant blood and lymphatic vessels, mean that GBCs easily invade the surrounding structures, and this is the most common method of GBC metastasis (4). The prognosis of GBC is poor, and the 5-year survival rate is extremely low ( 16\%) (5).

The treatment of GBC includes surgery, chemotherapy and palliative therapies (6). However, the cause of GBC has not previously been clear. CD74 molecule (CD74), also known as Ii or the invariant chain, is a type II transmembrane glycoprotein that has diverse immunological functions. The basic function of CD74 is associated with major histocompatibility complex (MHC) II, and CD74 is hypothesized to be associated with the processing of class II MHC molecules on antigen-presenting cells. In addition to antigen presentation, CD74 has also been identified as the receptor of macrophage migration inhibitory factor (MIF), which activates inflammation, and the expression of CD74 has been reported in numerous human malignant tumors, including breast, pancreatic and colorectal cancer (7-10). CD74 is important in carcinogenesis, as it acts as an accessory signaling molecule for cell proliferation (11). However, it is unknown whether CD74 is associated with the occurrence and development of GBC. The present study investigated the expression of CD74 in GBC tissues in order to elucidate the availability of CD74 as a novel biomarker for GBC. The associations between the level of CD74 expression the clinicopathological parameters, and epithelial growth factor receptor (EGFR), a known participant in numerous malignant diseases, were analyzed. CD74 was indicated to potentially be a core factor in the progression of GBC.

\section{Materials and methods}

Patients. A total of 54 patients who were diagnosed histopathologically with GBC underwent tumor resection at the Renmin Hospital of Wuhan University (Wuhan, China) between July 2009 and July 2013. The mean age of the patients was 61.2 years old and none of the patients received pre-operative or intraoperative chemotherapy or radiotherapy. The GBC 
tissues were divided into two groups; one of which was used to create paraffin-embedded tissue sections and the other of which was placed in liquid nitrogen for western blot analysis. The clinicopathological data of the patients were collected in order to analyze the association between the expression levels of CD74 and the clinicopathological data. All experiments using human tissues were reviewed and approved by the Committee for Ethical Reviews of Research involving Human Subjects of Remin Hospital of Wuhan University, and performed according to the Declaration of Helsinki.

Immunohistochemistry (IHC). The paraffin-embedded tissues were cut into $3-\mu \mathrm{m}$ thick tissue sections. To examine the expression of CD74 and EGFR, immunohistochemical analysis was performed using a standard streptavidin-peroxidase staining method. Briefly, the sections were deparaffinized and dehydrated using a graded series of ethanol solutions. The microscope slides were immersed in $10 \mathrm{mM}$ citrate buffer (pH 6.0; catalog no., G1202; Wuhan Goodbio Biotechnology Co., Ltd., Wuhan, China) and boiled for $5 \mathrm{~min}$ at $121^{\circ} \mathrm{C}$ in the pressure cooker (Supor YS20ED; Supor, Hangzhou, China) for the antigen retrieval. The slides were allowed to cool at room temperature. Hydrogen peroxide $(0.3 \%$; catalog no., H44023919; Guangdong Heng Jian Pharmaceutical Co., Ltd., Jiangmen, China) was used to halt the endogenous peroxidase activity for $15 \mathrm{~min}$ at room temperature. Non-specific binding was blocked using goat serum (5\%; catalog no., C0265; Beyotime Institute of Biotechnology, Haimen, China) for $10 \mathrm{~min}$. The primary antibodies used were a mouse anti-human anti-CD74 monoclonal antibody (dilution, 1:150; catalog no., ab9514; Abcam, Cambridge, UK) and a rabbit anti-human anti-EGFR monoclonal antibody (dilution, 1:100; catalog no., ZA0505; ZSGB-BIO, Beijing, China). Sections were incubated with the primary antibodies overnight at $4^{\circ} \mathrm{C}$, and then incubated with secondary antibodies from the UltraSensitiveTM SP (Mouse/Rabbit) IHC kit (catalog no., SP-9000, ZSGB-Bio). The staining results were visualized using 3,5-diaminobenzidine (DAB; catalog no., G1211; Wuhan Goodbio Biotechnology Co., Ltd.). For each immunohistochemical analysis, phosphate-buffered saline (PBS) was used instead of the primary antibody for the negative control.

Evaluation of the immunohistochemical findings. Two independent observers with IHC experience blindly evaluated the results. The BX53 upright microscope (Olympus Corporation, Tokyo, Japan) was used to capture images of the immunohistochemical staining results. Image-Pro Plus version 6.0 software (Media Cybernetics, Inc., Rockville, MD, USA) was used to judge the area and density of the dyed region, and the integrated optical density (IOD) value of the IHC section. According to the staining intensity, the results of IHC were assigned a score as follows: No dye, 0 ; pale yellow dye, 1; yellow dye, 2; and brown dye, 3 . The percentage of tumor cells was determined by taking an average score of at least 5 regions under x200 magnification. The mean percentage was then divided into four scored categories: $<5 \%$ tumor cells, $0 ; 5-25 \%$ tumor cells, 1 ; 26-50\% tumor cells, 2 ; and $51-100 \%$ tumor cells, 3 . Finally the score for staining intensity and the tumor cell percentage were added together as follows: An overall score between 0-2 was defined as negative expression; and an overall score between 3-6 was defined as positive expression (12).

Western blot analysis. The fresh GBC tissues were homogenized in ice-cold lysis buffer (Wuhan Goodbio Biotechnology Co., Ltd.) in the presence of protease inhibitor cocktail (catalog no., G2006; Wuhan Goodbio Biotechnology Co., Ltd.). The concentrations of protein in the samples were determined using the Bradford method (13) with bovine serum albumin (Wuhan Goodbio Biotechnology Co., Ltd.) as a standard. In brief, equal amounts of protein sample were separated on $10 \%$ sodium dodecyl sulfate polyacrylamide gels and then transferred to a polyvinylidene difluoride membrane. The membrane was blocked with 5\% skimmed milk in TBST (Tris-buffered saline containing $0.1 \%$ Tween-20) at room temperature for $2 \mathrm{~h}$ and then incubated with the rabbit anti-human anti-CD74 polyclonal antibody (1:1,000; Abcam) $4^{\circ} \mathrm{C}$ overnight. Subsequent to extensive rinsing with TBST (3 washes for $10 \mathrm{~min}$ each), the blots were incubated with IRDye $800 \mathrm{CW}$ goat anti-rabbit polyclonal secondary antibody (dilution, 1:10,000; catalog no., 926-32211; LI-COR Biosciences, Lincoln, NE, USA) at room temperature for $1.5 \mathrm{~h}$, and then the expression of CD74 was detected using the Odyssey CLx infrared imaging system (LI-COR Biosciences).

Statistical analysis. Data were analyzed with SPSS statistical software version 19.0 (IBM SPSS, Armonk, NY, USA). The $\chi^{2}$ test was used to assess the association between the expression of CD74 and the clinicopathological characters. The correlation between CD74 and EGFR was evaluated using the Spearman's rank correlation coefficient. $\mathrm{P}<0.05$ was considered to indicate a statistically significant difference.

\section{Results}

Immunostaining in normal and cancerous tissues, as determined by IHC. The positive expression of CD74 was indicated by yellow or brown staining in the cytoplasm. In the normal gallbladder tissues, the expression of CD74 was absent. In the GBC tissues, CD74 was more strongly stained in the poorly-differentiated carcinoma compared with the well-differentiated tissues (Fig. 1).

Expression of CD74 in GBC tissues, as determined by western blot analysis. The expression of CD74 was detected in tissue samples taken immediately following resection of GBC tumors, and the western blotting results revealed that in the poorly-differentiated GBC tissues, CD74 was upregulated compared with the moderately- and well-differentiated GBC tissues. The outcome of the western blot analysis was consistent with the immunohistochemical findings (Fig. 2).

Correlation between CD74 expression and clinicopathological characteristics. The correlation between CD74 expression and the clinicopathological characteristics of GBC are shown in Table I (14). In the GBC tissues, the poorly-differentiated carcinomas exhibited increased CD74(+) expression compared with the well- and moderately-differentiated carcinomas, the tumor-node-metastasis (TNM) stage II-IV tissues exhibited increased CD74(+) expression compared with the TNM 
Table I. Correlation between CD74 expression and the clinicopathological characteristics of gallbladder carcinoma.

\begin{tabular}{|c|c|c|c|c|c|}
\hline \multirow[b]{2}{*}{ Clinical features } & \multirow[b]{2}{*}{ No. of patients $(\mathrm{n}=54)$} & \multicolumn{2}{|c|}{ CD74 expression, $\mathrm{n}$} & \multirow[b]{2}{*}{$\chi^{2}$ value } & \multirow[b]{2}{*}{ P-value } \\
\hline & & + & - & & \\
\hline Gender & & & & 0.087 & 0.768 \\
\hline Male & 17 & 12 & 5 & & \\
\hline Female & 37 & 23 & 14 & & \\
\hline Age, years & & & & 0.055 & 0.814 \\
\hline$<61$ & 23 & 14 & 9 & & \\
\hline$\geq 61$ & 31 & 21 & 10 & & \\
\hline Differentiation & & & & 8.277 & 0.016 \\
\hline Well & 8 & 2 & 6 & & \\
\hline Moderate & 28 & 18 & 10 & & \\
\hline Poor & 18 & 15 & 3 & & \\
\hline Depth of tumor infiltration & & & & 8.406 & 0.004 \\
\hline $\mathrm{T}_{\mathrm{IS}}-\mathrm{T}_{2}$ & 24 & 10 & 14 & & \\
\hline $\mathrm{T}_{3}-\mathrm{T}_{4}$ & 30 & 25 & 5 & & \\
\hline Lymph-node metastasis & & & & 0.549 & 0.459 \\
\hline No & 29 & 17 & 11 & & \\
\hline Yes & 25 & 12 & 13 & & \\
\hline TNM stage & & & & 4.853 & 0.028 \\
\hline $0-\mathrm{I}$ & 5 & 1 & 4 & & \\
\hline II-IV & 49 & 34 & 15 & & \\
\hline
\end{tabular}

CD74, cluster of differentiation; TNM, tumor-node-metastasis; $\mathrm{T}_{\text {IS }}$, carcinoma in situ.
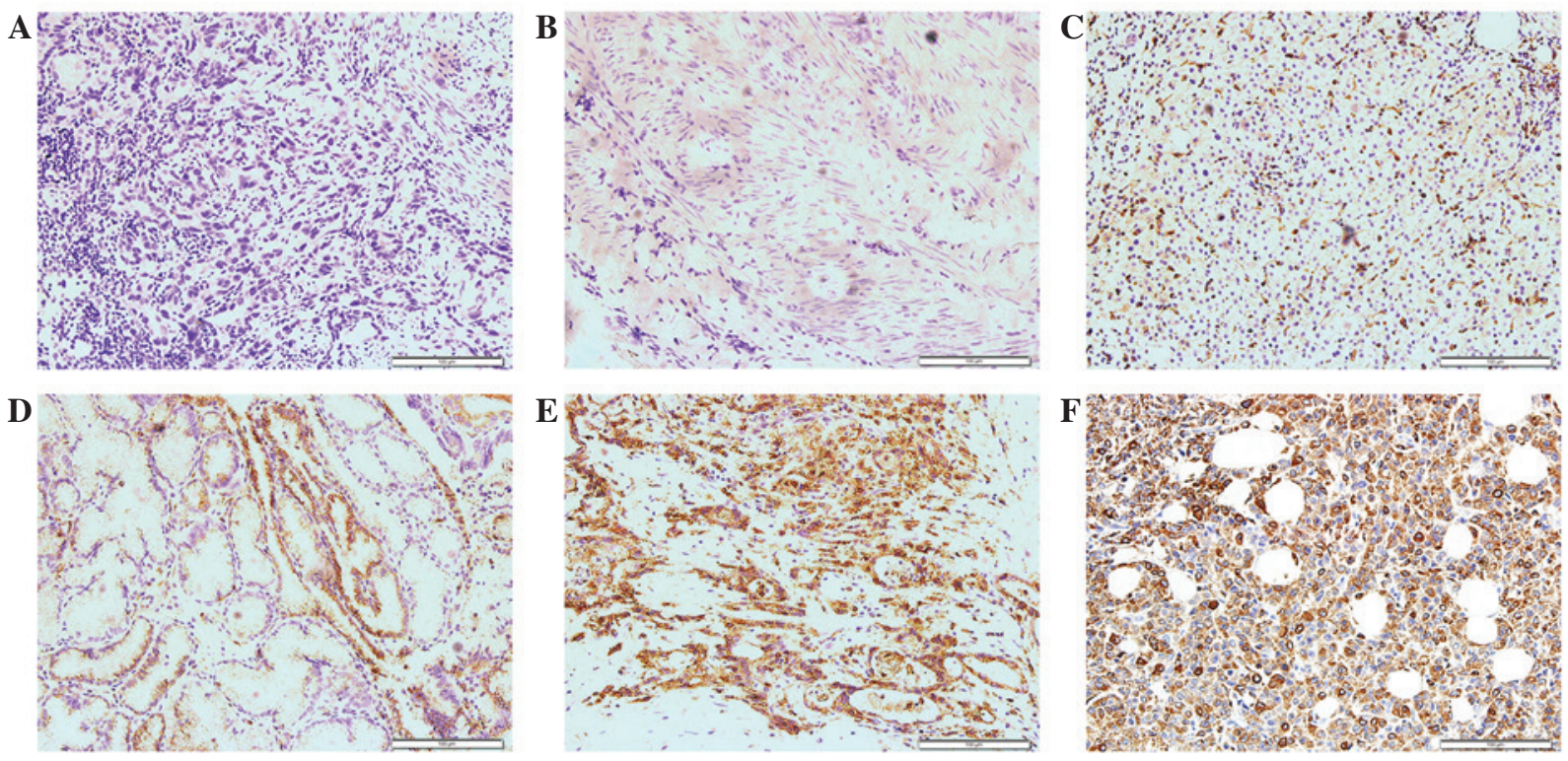

Figure 1. Expression of cluster of differentiation 74 in normal gallbladder and GBC tissues (strepavidin-peroxidase; magnification, x200; scale bar, $100 \mu \mathrm{m}$.) (A) Negative control; (B) normal gallbladder tissue; (C) well-differentiated type of GBC; (D) moderately-differentiated type of GBC; (E) poorly-differentiated type of GBC; and (F) positive control (colon carcinoma). GBC, gallbladder carcinoma.

stage 0 -II tissues, and the group of tissues with increased depth of tumor infiltration, $\mathrm{T}_{3}-\mathrm{T}_{4}$, exhibited increased CD74(+) expression compared with the $\mathrm{T}_{\mathrm{is}} \mathrm{T}_{2}$ group. There were no significant differences in the expression of CD74 for certain clinical features, including gender, age and lymph node metastasis.
Correlation between CD74 expression and EGFR expression in $G B C$. The expression and IOD values of EGFR were examined in the GBC tissues. EGFR expression in GBC tissues may be observed in Fig. 3. The IOD values of EGFR are presented in Fig. 4A. Using the Spearman's rank correlation coefficient, 


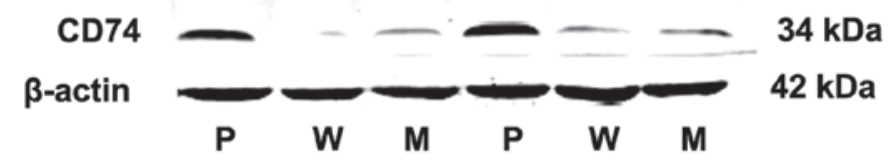

Figure 2. Western blotting to detect the expression of CD74 in GBC. P, poorly-differentiated GBC; M, moderately-differentiated GBC; W, well-differentiated GBC; CD74, cluster of differentiation 74; GBC, gallbladder carcinoma.
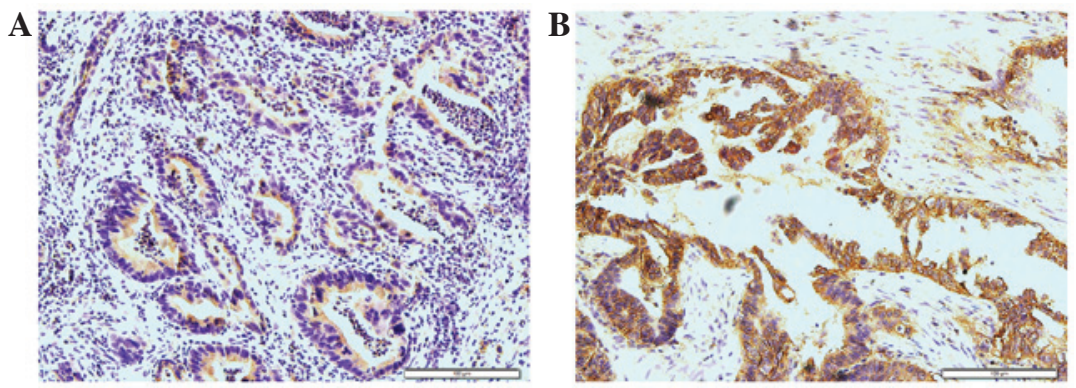

Figure 3. Expression of epithelial growth factor receptor in gallbladder carcinoma. (A) Low expression and (B) high expression (strepavidin-peroxidase; magnification, x200; scale bar, $100 \mu \mathrm{m}$.).
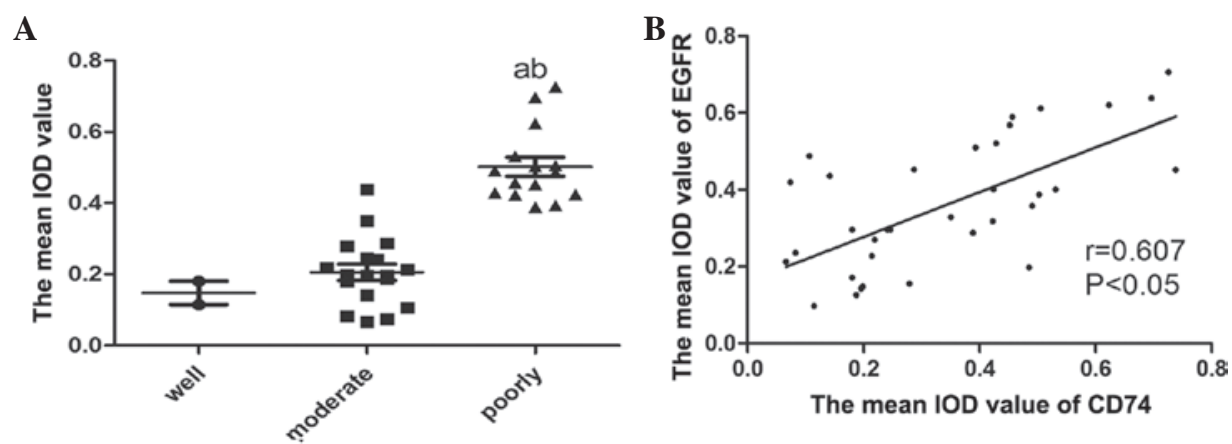

Figure 4. Mean IOD value of CD74 and its correlation to EGFR. (A) Mean IDO value of various gallbladder carcinoma tissues. ${ }^{a} \mathrm{P}<0.05$, poorly vs. well; ${ }^{\mathrm{b}} \mathrm{P}<0.05$, poorly vs. moderate. (B) Correlation of the mean IOD of CD74 and EGFR, as determined by Spearman's rank correlation coefficient ( $\mathrm{r}=0.607$; $\mathrm{P}<0.05$ ). IOD, integrated optical density; CD74, cluster of differentiation 74; EGFR, epithelial growth factor receptor; poorly, poorly-differentiated; well, well-differentiated; moderate, moderately-differentiated.

the IOD values of CD74 were shown to be positively correlated with the levels of EGFR (Fig. 4B). The tissue sections that exhibited the expression of CD74 strongly tended to be associated with the increased expression of EGFR.

\section{Discussion}

GBC is the seventh most common cancer of the digestive system (15). The early diagnosis of GBC occurs infrequently due to the lack of specific clinical characteristics associated with the disease, and as GBC is challenging to distinguish from common digestive tract diseases, including cholelithiasis and chronic cholecystitis. Therefore, the majority of GBC patients are in the advanced stages of the disease by the time they arrive at hospital for treatment. The advanced stages of $\mathrm{GBC}$ are associated with a poor prognosis and high mortality rate; the mean survival rate of patients with advanced GBC is $\sim 6$ months and the 5 -year survival rate is $<32 \%(7,16)$. Therefore, finding a novel tumor biomarker that may be used for the early diagnosis of GBC may have a positive effect on the 5-year survival rate of GBC. Numerous clinical studies have suggested that CD74 is important in the pathogenesis of hematological malignancies and various solid carcinomas (17). The present study reported the expression of CD74 and EGFR in GBC by immunohistochemical staining, and used Spearman's rank correlation coefficient in order to study the association between the expression of CD74 and EGFR in GBC. The results revealed that CD74 was overexpressed in the GBC tissues, and that the mean staining intensity of CD74 in the poorly-differentiated GBC tissues was increased compared with that in the well- and moderately-differentiated tissues. The results of the western blot analysis also showed that the expression of CD74 was upregulated in the poorly-differentiated GBC tissues. The precise role of CD74 in GBC has not been previously elucidated. The study by Roche and Cresswell (18) reported that CD74 is the invariant chain of MHC-II and a type of transmembrane glycoprotein. CD74 is expressed at high levels in antigen-presenting cells, including B-cells, monocytes and macrophages, so it is extremely important in antigen presentation. D-related (DR)-CD74 complexes have 
a suppressive effect on the host immune response, which results in the promotion of tumor proliferation $(19,20)$. Certain studies have suggested that the overexpression of CD74 in malignancies may block endogenous tumor antigen presentation by MHC-II, resulting in immune escape in vivo (21). In B-cell lymphoma and multiple myeloma, CD74 has been reported as a novel and promising therapeutic target $(22,23)$. In non-hematological malignancies, including gastric carcinoma, renal cancer and non-small cell lung cancer, CD74 has also been reported as a novel biomarker for its value in predicting prognoses (24-26).

In the present study, the mean IOD value of CD74 in the poorly-differentiated GBC tissues was increased compared with the well- and moderately-differentiated tissues, and the western blotting results were consistent with this finding. These results indicated that CD74 may take part in the process of differentiation in GBC tumor cells. The role of CD74 in carcinogenesis may be associated with MIF, which is an important inflammatory cytokine and has a strong association with carcinogenesis (27). CD74 is the receptor of MIF, and the MIF and CD74 ligand/receptor combination may be a key factor in determining the difference between carcinogenesis and chronic inflammation. The ligand/receptor combination may increase the proliferation of epithelial cells through the tumor protein p53 (p53) pathway (28). p53 is a tumor suppressor, and when $\mathrm{p} 53$ is blocked from the cytomembrane to the nucleus, the function of the apoptotic pathway is decreased and proliferation is increased (29). The MIF and CD74 combination may also increase the proliferation of epithelial cells by activating EGFR, which has been established as a biomarker and therapeutic target in various solid tumors and is important in the proliferation, migration and invasion of tumor cells (30). The combination may also upregulate the pro-inflammatory cytokine interleukin-8, which then binds to a receptor on the surface of the epithelial cell. This novel ligand/receptor combination may regulate the level of $\operatorname{EGFR}(31,32)$. The present study detected the expression of EGFR and CD74 by IHC within the same section of GBC tissue, and the mean IOD value of CD74 and EGFR was tested using the Spearman's rank correlation coefficient. The result revealed that the IHC staining of CD74 positively correlated with EGFR in the GBC tissues. The samples that exhibited an increased expression level of CD74 were associated with an increased expression level of EGFR.

In conclusion, the IHC and western blotting results of CD74 revealed that $\mathrm{CD} 74$ was closely associated with the degree of differentiation in the GBC tissues, and that the correlation between CD74 and EGFR, as determined by Spearman's rank correlation coefficient, was positive. As the present study did not examine the expression of CD74 in GBC at the RNA and cellular levels, additional studies are required to determine the precise role of CD74 in GBC. The present results elucidate the potential role of CD74 as a key participator in the progression of GBC.

\section{Acknowledgements}

The present study was supported by the National Natural Science Foundation of China (grant no. 81370562) and the Projects of Medical and Health Technology Development
Program in Shandong Province (grant no. 2013GGB14096). The authors would like to thank Dr. Yongfei Tang of the Department of Pathology, Renmin Hospital of Wuhan University (Wuhan, China) for assistance with the evaluation of immunohistochemical findings.

\section{References}

1. Hundal R and Shaffer EA: Gallbladder cancer: Epidemiology and outcome. Clin Epidemiol 6: 99-109, 2014.

2. Lazcano-Ponce EC, Miquel JF, Muñoz N, Herrero R, Ferrecio C, Wistuba II, Alonso de Ruiz P, Aristi Urista G and Nervi F: Epidemiology and molecular pathology of gallbladder cancer. CA Cancer J Clin 51: 349-364, 2001.

3. Kaushik SP: Current perspectives in gallbladder carcinoma. J Gastroenterol Hepatol 16: 848-854, 2001.

4. Gore RM and Shelhamer RP: Biliary tract neoplasms: Diagnosis and staging. Cancer Imaging 7: S15-S23, 2007.

5. Carriaga MT and Henson DE: Liver, gallbladder, extrahepatic bile ducts and pancreas. Cancer 75 (Suppl 1): S171-S190, 1995.

6. Wernberg JA and Lucarelli DD: Gallbladder cancer. Surg Clin North Am 94: 343-360, 2014.

7. Mastoraki A, Papanikolaou IS, Konstandiadou I, Sakorafas G and Safioleas M. Facing the challenge of treating gallbladder carcinoma. Review of the literature. Hepatogastroenterology 57: 215-219, 2010.

8. Tassi E, Braga M, Longhi R, Gavazzi F, Parmiani G, Di Carlo V and Protti MP: Non-redundant role for IL-12 and IL-27 in modulating Th2 polarization of carcinoembryonic antigen specific CD4 T cells from pancreatic cancer patients. PloS One 4: e7234, 2009.

9. Zhang JF, Hua R, Liu DJ, Liu W, Huo YM and Sun YW: Effect of CD74 on the prognosis of patients with resectable pancreatic cancer. Hepatobiliary Pancreat Dis Int 13: 81-86, 2014.

10. Kindt N, Lechien JR, Nonclercq D, Laurent G and Saussez S: Involvement of CD74 in head and neck squamous cell carcinomas. J Cancer Res Clin Oncol 140: 937-947, 2014.

11. Greenwood C, Metodieva G, Al-Janabi K, Lausen B, Alldridge L, Leng L, Bucala R, Fernandez N and Metodiev MV: Stat1 and CD74 overexpression is co-dependent and linked to increased invasion and lymph node metastasis in triple-negative breast cancer. J Proteomics 75: 3031-3040, 2012.

12. Pinheiro C, Longatto-Filho A, Scapulatempo C, Ferreira L, Martins S, Pellerin L, Rodrigues M, Alves VA, Schmitt F and Baltazar F: Increased expression of monocarboxylate transporters 1, 2 and 4 in colorectal carcinomas. Virchows Arch 452: 139-146, 2008.

13. Bradford MM: A rapid and sensitive method for the quantitation of microgram quantities of protein utilizing the principle of protein-dye binding. Anal Biochem 72: 248-254. 1976.

14. Li J, Liu W, Qu Q, Hong T, Xu X, Li B, Wang Y and He X. Clinical assessment using TNM staging for 151 patients with gallbladder carcinoma. Chinese Journal of Hepatobiliary Surgery 20: 507-510, 2014 (In Chinese and English).

15. Reddy SK and Clary BM: Surgical management of gallbladder cancer. Surg Oncol Clin N Am 18: 307-324, 2009.

16. Stinton LM and Shaffer EA: Epidemiology of gallbladder disease: Cholelithiasis and cancer. Gut Liver 6: 172-187, 2012.

17. Nagata S, Jin YF, Yoshizato K, Nagata S, Jin YF, Yoshizato K, Tomoeda M, Song M, Iizuka N, Kitamura M, Takahashi H, Eguchi $\mathrm{H}$, Ohigashi $\mathrm{H}$, et al. CD74 is a novel prognostic factor for patients with pancreatic cancer receiving multimodal therapy. Ann Surg Oncol 16: 2531-2538, 2009.

18. Roche PA and Cresswell P: Invariant chain association with HLA-DR molecules inhibits immunogenic peptide binding. Nature 345: 615-618, 1990.

19. Hippo Y, Yashiro M, Ishii M, Taniguchi H, Tsutsumi S, Hirakawa K, Kodama T and Aburatani H: Differential gene expression profiles of scirrhous gastric cancer cells with high metastatic potential to peritoneum or lymph nodes. Cancer Res 61: 889-895, 2001.

20. Jiang $\mathrm{Z}, \mathrm{Xu} \mathrm{M}$, Savas L, LeClair $\mathrm{P}$ and Banner BF: Invariant chain expression in colon neoplasms. Virchows Arch 435: 32-36, 1999.

21. Xu M, Qiu G, Jiang Z, von Hofe E and Humphreys RE: Genetic modulation of tumor antigen presentation. Trends Biotechnol 18: 167-172, 2000. 
22. Griffiths GL, Mattes MJ, Stein R, Govindan SV, Horak ID, Hansen HJ and Goldenberg DM: Cure of SCID mice bearing human B-lymphoma xenografts by an anti-CD74 antibody-anthracycline drug conjugate. Clin Cancer Res 9: 6567-6571, 2003.

23. Burton JD, Ely S, Reddy PK, Stein R, Gold DV, Cardillo TM and Goldenberg DM: CD74 is expressed by multiple myeloma and is a promising target for therapy. Clin Cancer Res 10: 6606-6611, 2004.

24. Ioachim HL, Pambuccian SE, Hekimgil M, Giancotti FR and Dorsett BH: Lymphoid monoclonal antibodies reactive with lung tumors. Diagnostic applications. Am J Surg Pathol 20: 64-71, 1996.

25. Lazova R, Moynes R, May D and Scott G: LN-2 (CD74). A marker to distinguish atypical fibroxanthoma from malignant fibrous histiocytoma. Cancer 79: 2115-2124, 1997.

26. Young AN, Amin MB, Moreno CS, Lim SD, Cohen C, Petros JA, Marshall FF and Neish AS: Expression profiling of renal epithelial neoplasms: A method for tumor classification and discovery of diagnostic molecular markers. Am J Pathol 158: $1639-1651,2001$

27. Sanchez-Niño MD, Sanz AB, Ruiz-Andres O, Poveda J Izquierdo MC, Selgas R, Egido J and Ortiz A. MIF, CD74 and other partners in kidney disease: Tales of a promiscuous couple. Cytokine Growth Factor Rev 24: 23-40, 2013.
28. Beswick EJ, Pinchuk IV, Suarez G, Sierra JC and Reyes VE: Helicobacter pylori CagA-dependent macrophage migration inhibitory factor produced by gastric epithelial cells binds to CD74 and stimulates procarcinogenic events. J Immunol 176: 6794-6801, 2006.

29. Jung H, Seong HA and Ha H: Critical role of cysteine residue 81 of macrophage migration inhibitory factor (MIF) in MIF-induced inhibition of p53 activity. J Biol Chem 283: 20383-20396, 2008.

30. Maharshak N, Cohen S, Lantner F, Hart G, Leng L, Bucala R and Shachar I. CD74 is a survival receptor on colon epithelial cells. World J Gastroenterol 16: 3258-3266, 2010.

31. Beswick EJ and Reyes VE: Macrophage migration inhibitory factor and interleukin- 8 produced by gastric epithelial cells during Helicobacter pylori exposure induce expression and activation of the epidermal growth factor receptor. Infect Immun 76: 3233-3240, 2008

32. Itoh Y, Joh T, Tanida S, Sasaki M, Kataoka H, Itoh K, Oshima T, Ogasawara N, Togawa S, Wada T, et al: IL-8 promotes cell proliferation and migration through metalloproteinase-cleavage proHB-EGF in human colon carcinoma cells. Cytokine 29: 275-282, 2005. 